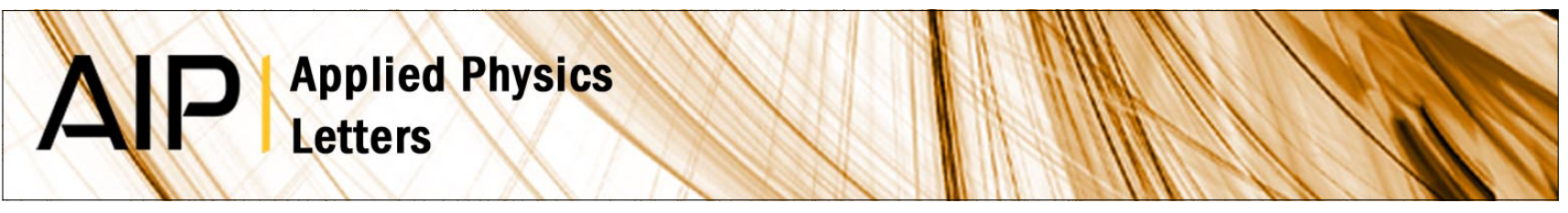

\title{
Surface rippling on bulk metallic glass under nanosecond pulse laser ablation
}

Y. Liu, M. Q. Jiang, G. W. Yang, Y. J. Guan, and L. H. Dai

Citation: Appl. Phys. Lett. 99, 191902 (2011); doi: 10.1063/1.3656700

View online: http://dx.doi.org/10.1063/1.3656700

View Table of Contents: http://apl.aip.org/resource/1/APPLAB/v99/i19

Published by the American Institute of Physics.

\section{Related Articles}

Melting of monatomic glass with free surfaces

J. Chem. Phys. 136, 104506 (2012)

Molecular packing in highly stable glasses of vapor-deposited tris-naphthylbenzene isomers

J. Chem. Phys. 136, 094505 (2012)

Role of aluminum as an oxygen-scavenger in zirconium based bulk metallic glasses

Appl. Phys. Lett. 100, 071909 (2012)

Prediction of glass-forming ability and characterization of atomic structure of the Co-Ni-Zr metallic glasses by a proposed long range empirical potential

J. Appl. Phys. 111, 033521 (2012)

Structurally determined directionality identifies the boundary between mobile and immobile domains in a disordered material

J. Chem. Phys. 136, 054507 (2012)

\section{Additional information on Appl. Phys. Lett.}

Journal Homepage: http://apl.aip.org/

Journal Information: http://apl.aip.org/about/about_the_journal

Top downloads: http://apl.aip.org/features/most_downloaded

Information for Authors: http://apl.aip.org/authors

\section{ADVERTISEMENT}

NEW!

iPeerReview

AIP's Newest App

$\frac{\Delta I P}{L}$
Authors...

Reviewers...

Check the status of

submitted papers remotely!

AIP Publishing 


\title{
Surface rippling on bulk metallic glass under nanosecond pulse laser ablation
}

\author{
Y. Liu, ${ }^{1}$ M. Q. Jiang, ${ }^{1}$ G. W. Yang, ${ }^{2}$ Y. J. Guan, ${ }^{1}$ and L. H. Dai ${ }^{1, a)}$ \\ ${ }^{1}$ State Key Laboratory of Nonlinear Mechanics, Institute of Mechanics, Chinese Academy of Sciences, \\ Beijing 100190, China \\ ${ }^{2}$ State Key Laboratory of Optoelectronic Materials and Technologies, School of Physics and Engineering, \\ Zhongshan University, Guangzhou, Guangdong 510275, China
}

(Received 31 August 2011; accepted 10 October 2011; published online 8 November 2011)

\begin{abstract}
We report an interesting surface ripple patterns in the irradiated area of a Zr-based bulk metallic glass by nanosecond pulse laser with single shot. Such surface rippling phenomenon can be ascribed to the Kelvin-Helmholtz instability at the interface between the molten layer and the expanding plasma plume. The analytical instability criterion is obtained via a perturbation analysis. Furthermore, the model demonstrates that the characteristic spacing of the ripples is dominated by the density, transverse velocity of the plasma wind, and the surface tension of the molten layer. The predicted spacing agrees well with the experimentally observed values. The results are fundamentally useful for laser-processing bulk metallic glasses (BMGs) and even for understanding the nature of flow in BMGs. (C) 2011 American Institute of Physics. [doi:10.1063/1.3656700]
\end{abstract}

Since the first operating devices in $1960,{ }^{1}$ high-power laser has captured much attention of the scientific and engineering community. In the course of practical applications, ${ }^{2}$ the laser-induced surface patterns open a window into understanding the mechanism for the laser-material interactions. ${ }^{3-7}$ It is noted that, however, previous works mainly focused on crystalline materials (metals, alloys, semiconductor, etc.) or traditional glassy materials. Bulk metallic glasses (BMGs) with both glassy-state structure and metallic-bonding character show a series of intriguing mechanical, physical, and chemical properties, having wide range functional and structural applications. ${ }^{8-12}$ Due to rapid heating and cooling, lasers have been widely used to welding, cutting, cladding, alloying, glazing, annealing, melting, or ablating the small-scaled amorphous alloys such as ribbons, films, and wires, ${ }^{13-17}$ very recently extending to BMGs. ${ }^{18,19}$ Through laser-processing, microstructures, ${ }^{15-17}$ magnetic properties, ${ }^{14}$ forming ability, ${ }^{20}$ and mechanical properties ${ }^{19}$ of this kind of glassy alloys can be significantly improved, whereas only few works have focused on the surface patterns. ${ }^{13,21,22}$ Its underlying physics is still unclear and deserves further investigations. In this letter, we observed a concentric surface ripples on a typical $\mathrm{Zr}$ based BMG irradiated by single pulsed nanosecond laser. The possible mechanism of such rippling phenomenon is quantitatively discussed in terms of the hydrodynamic instability.

A typical BMG $\mathrm{Zr}_{41.2} \mathrm{Ti}_{13.8} \mathrm{Cu}_{10} \mathrm{Ni}_{12.5} \mathrm{Be}_{22.5}$ (Vit 1) was adopted as target materials. Target specimens $(10 \mathrm{~mm} \times 10 \mathrm{~mm} \times 2 \mathrm{~mm})$ were cut by wire electrical discharge machining the as-cast materials. Then, the target surfaces were electro-polished. The glassy nature of the targets was confirmed by x-ray diffraction (XRD). In order to examine the response of the original structure of Vit $1 \mathrm{BMG}$ to the laser, single pulse laser ablation experiments were performed using a Q-switched Nd: YAG solid state laser (wavelength as $532 \mathrm{~nm}$, pulse duration as $10 \mathrm{~ns}$, and maximum

\footnotetext{
a) Author to whom correspondence should be addressed. Electronic mail: lhdai@lnm.imech.ac.cn.
}

pulse energy as $500 \mathrm{~mJ}$ ) in ambient air and at room temperature. After irradiation, a high-resolution scanning electron microscope was used to examine the surface morphology of all targets.

Figure 1(a) displays the typical surface features of the Vit 1 BMGs irradiated by single pulse laser with the intensity of about $2.5 \times 10^{13} \mathrm{~W} / \mathrm{m}^{2}$. As marked by " $\mathrm{A}$ " in Fig. 1(a), there exists a concentrated ablated region (about $200 \mu \mathrm{m}$ diameter) at the center of the irradiated area. Fig. 1(b) shows a highmagnification micrograph of this ablated region, where the ablation-induced plasma plume would occur due to the pulse energy higher over the ablation threshold of material. A closeup view of the area " $B$ " in Fig. 1(a) is presented in Fig. 1(c). An enlarged view of the area " $C$ " in Fig. 1(c) is presented in Fig. 1(d). Figures 1(e) and 1(f) correspond to the areas "D" and " $E$ " in Fig. 1(d), respectively. It is noted from these figures that the material undergoes melting in the region from the outer boundary of the central ablated region to the edge of the irradiated area. Quite interestingly, concentric ripples at a microscale are prone to form prior to the material solidification. The characteristic spacing of the ripples is approximately about $5 \mu \mathrm{m}$ (see Figs. 2(d) and 2(e)). As indicated in Fig. 2(f), the ripple spacing decreases at the edge of the irradiation area due to the solid-boundary effect. The observed spacing values are much greater than the incident laser wavelength $(532 \mathrm{~nm})$. This implies that the light interference effect ${ }^{7}$ should be excluded, whereas a certain hydrodynamic mechanism should become operative. ${ }^{6}$ The large scaled ripple-type surface patterns look much like an unstable interface between the wind and the water in a pond when the wind skitters along the water surface. In the present case, the molten Vit 1 at the irradiated area plays the role of "water," and the "wind" comes from expansion of the high pressure plasma plume generated at the central ablated region. The laser-induced plasma plume and the molten Vit 1 pool constitute two fluid layers with different density and horizontal velocity. In hydrodynamics, this kind of configuration turns our attention to the Kelvin-Helmholtz (K-H) instability. ${ }^{23}$ We therefore identify the K-H instability 


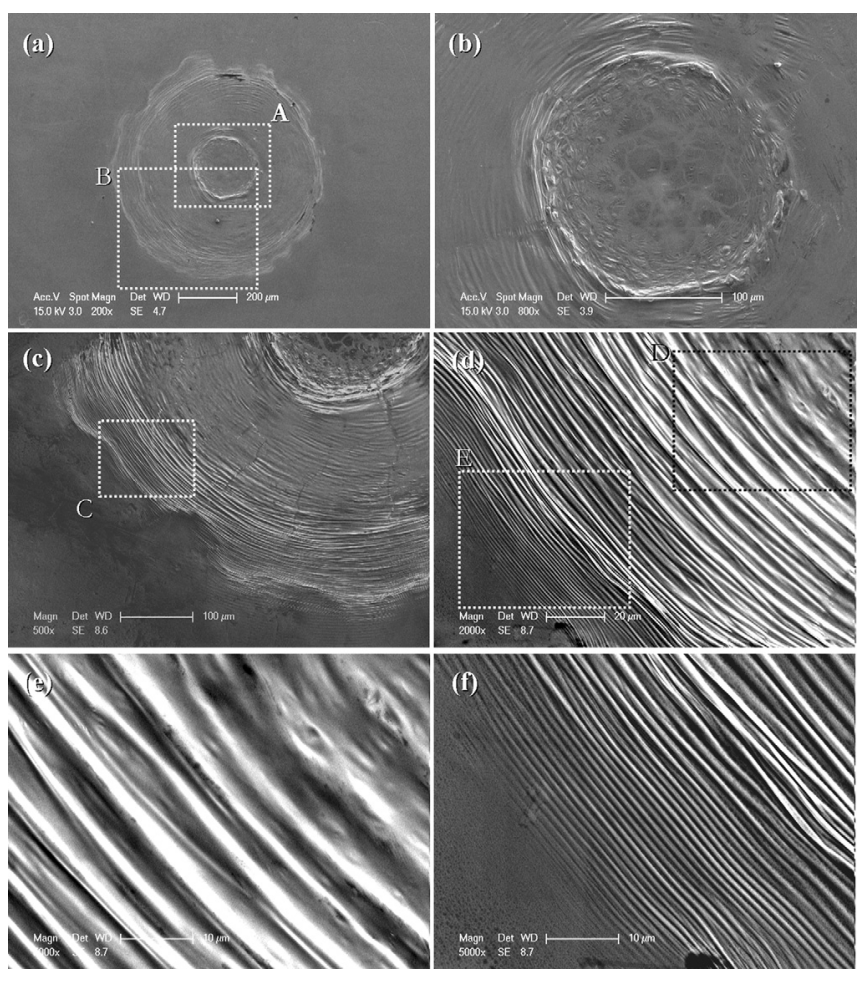

FIG. 1. The irradiated morphology of the Vit 1 BMG by a nanosecond pulse laser with singe shot. (a) The full view of irradiated area. (b) The concentrated ablated region marked by "A" in (a). (c) A close-up view of the area "B" in (a). (d) An enlarged view of the area "C" in (c). (e) and (f) correspond to the areas "D" and "E" in (d), respectively.

as a viable mechanism if the predicted instability wavelength is consistent with the characteristic spacing of the observed ripples.

The relative motion of two layers of fluids, i.e., the Vit 1 melt and the plasma plume, is modeled in an Eulerian coordinate system $(x, z)$. The incident direction of the laser is along the negative $z$-axis. The expanding plume is located in region $\mathrm{I}, z>0$; the melted Vit 1 in region $\mathrm{II}, z<0$. The initial interface is at $z=0$. To be specific, we suppose that the streaming only takes place in the $x$-direction. Both fluids are

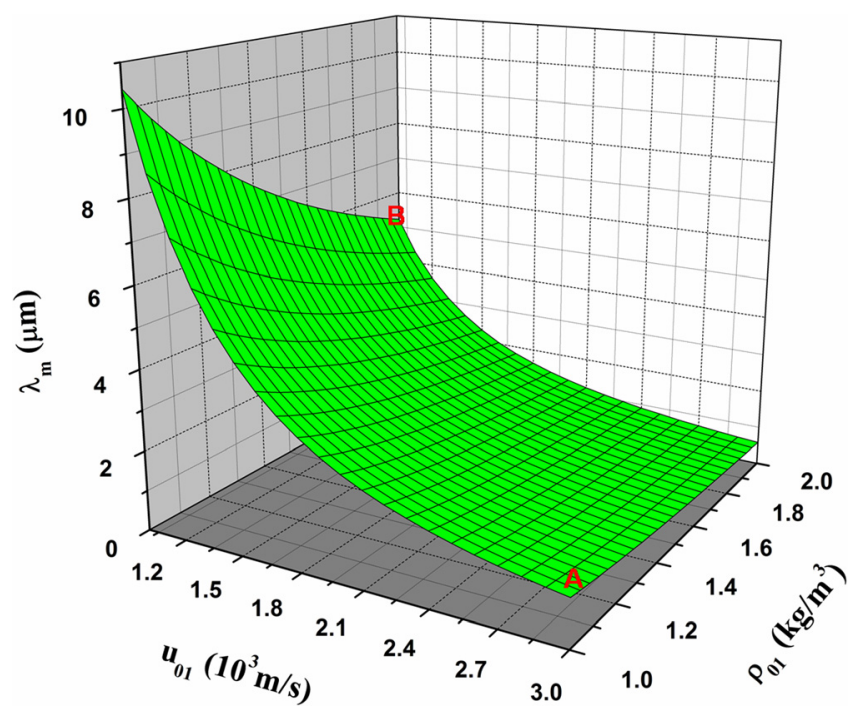

FIG. 2. (Color online) Prediction of ripple spacing $\lambda_{m}$ with the change of density $\rho_{01}$ and initial horizontal velocity $u_{01}$ of the expanding plasma. considered as incompressible and viscid, during which the effects of surface tension and gravity are involved. The governing equations for the full field including the upper plume, the lower melt and their interface are given by

$$
\begin{gathered}
\rho \mathrm{d} u / \mathrm{d} t=-\partial p / \partial x+\mu \nabla^{2} u \\
\rho \mathrm{d} w / \mathrm{d} t=-\partial p / \partial z-\rho g+\mu \nabla^{2} w+\sigma \delta\left(z-z_{s 0}\right) \partial^{2} z_{s} / \partial x^{2} \\
\mathrm{~d} \rho / \mathrm{d} t=0 \\
\nabla \cdot(u, w)=0 \\
\mathrm{~d} z_{s} / \mathrm{d} t=w_{s}
\end{gathered}
$$

where Eqs. (1) and (2) are the equation of motion along the $x$-direction and $z$-direction, respectively, Eq. (3) the incompressible condition, Eq. (4) the continuity equation, and Eq. (5) the interface continuity condition. In these equations, $\rho$ is the density, $u$ is the horizontal velocity, $w$ is the vertical velocity, $\mu$ denotes the coefficient of viscosity, $p$ is the pressure, $g$ is the gravity acceleration, $\sigma$ is the surface tension constant, $\delta$ is the Dirac delta function, the subscript " $s$ " denotes the interface, and $z_{s 0}=0$ is the initial position of the interface.

The K-H instability can be studied by imposing a perturbation $\left\{u^{\prime}, w^{\prime}, p^{\prime}, \rho^{\prime}, \mu^{\prime}, z_{s}^{\prime}\right\}$ of the form $\exp i(\omega t+k x)$ on the initial static state $\left\{u_{0}, w_{0}, p_{0}, \rho_{0}, \mu_{0}, z_{s 0}\right\}$ and then concentrating on the evolution of the disturbed interface. In order to highlight the essential physics, we assume that $u_{0}=u_{0}(z)$, $w_{0}=0, \rho_{0}=\rho_{0}(z)$, and $\mu_{0}=$ const. Here, $k$ is the wave number, and $\omega=\gamma+i \alpha$, here $\alpha$ is related to the rate of instability growth. The stability of the interface is now determined by the sign of $\alpha$. If $\alpha<0$, it is stable; in the opposite case, unstable.

Inserting the perturbation into Eqs. (1)-(5) and only considering the first order terms of the perturbation, we can obtain the governing equation for the perturbed velocity $w^{\prime}$,

$$
\begin{aligned}
D[- & \rho_{0} i\left(\omega+k u_{0}\right) D w^{\prime}+i \rho_{0} k D u_{0} w^{\prime} \\
& \left.+\mu_{0}\left(D^{2}-k^{2}\right) D w^{\prime}-i \mu^{\prime} k D^{2} u_{0}\right] \\
& +\rho_{0} i\left(\omega+i k u_{0}\right) k^{2} w^{\prime}-\mu_{0}\left(D^{2}-k^{2}\right) k^{2} w^{\prime} \\
= & g k^{2} z^{\prime} D \rho_{0}-\sigma k^{4} z^{\prime} \delta(z),
\end{aligned}
$$

where $D=\partial / \partial z$. Through the integral approximation, the boundary condition that is satisfied at a surface of discontinuity is obtained as follows:

$$
\begin{aligned}
\Delta_{s}[ & -\rho_{0} i\left(\omega+k u_{0}\right) D w^{\prime}+i \rho_{0} k D u_{0} w^{\prime} \\
& \left.+\mu_{0}\left(D^{2}-k^{2}\right) D w^{\prime}-i \mu^{\prime} k D^{2} u_{0}-\mu_{0} k^{2} D w^{\prime}\right] \\
= & g k^{2}\left[\Delta_{s}\left(\rho_{0}\right)-\sigma k^{2} / g\right] z_{s}^{\prime}
\end{aligned}
$$

where $\Delta_{s}=f_{z_{s 0}+0}-f_{z_{00}-0}$. Keep in mind that the domain of interest is the two thin fluid layers in the vicinity of the interface. Therefore, we shall further suppose that the two fluids are streaming with the constant velocities $u_{01}$ and $u_{02}$ as well 
as constant densities $\rho_{01}$ and $\rho_{02}$. In this case, we can obtain the solution of Eq. (6) except for the interface, that is,

$$
\begin{gathered}
w_{1}^{\prime}=z_{s}^{\prime} i\left(\omega+k u_{01}\right) e^{-k z} \quad(z>0), \\
w_{2}^{\prime}=z_{s}^{\prime} i\left(\omega+k u_{02}\right) e^{k z} \quad(z<0) .
\end{gathered}
$$

Applying the boundary condition (7) to the solutions, we have the spectral equation for the initial growth rate $\alpha$ of the perturbation,

$$
a_{4} \alpha^{4}+a_{3} \alpha^{3}+a_{2} \alpha^{2}+a_{1} \alpha+a_{0}=0 .
$$

The coefficients of this polynomial are determined by the initial static sate of liquids and the perturbation wave number. According to the Routh-Hurwitz criterion, ${ }^{24}$ the instability criterion for the interface between the upper plasma plume and the lower molten Vit 1, i.e., the onset condition for the K-H instability, can be obtained,

$$
\left(u_{01}-u_{02}\right)^{2}>\frac{\left(\mu_{01}+\mu_{02}\right)^{2}}{\rho_{01} \mu_{02}^{2}+\rho_{02} \mu_{01}^{2}}\left[\sigma k+\frac{g}{k}\left(\rho_{02}-\rho_{01}\right)\right] .
$$

It can be readily seen from this criterion that the difference in initial horizontal velocity $u_{01}-u_{02}$ incurs the interface instability; the surface tension $\sigma$, gravity $g$, and the density difference $\rho_{02}-\rho_{01}$ suppress the instability development. As for the viscosity, the increase in the ratio of the upper viscosity to lower viscosity $\mu_{01} / \mu_{02}$ facilitates the K-H instability. In other words, the larger the plasma viscosity is, and the smaller the melt viscosity is, the easier their interface instability occurs.

Based on the instability criterion (11), the dynamic balance between the stabilizing and destabilizing effects determines a critical wavelength,

$$
\lambda_{c}=\frac{\left(\mu_{01}+\mu_{02}\right)^{2}}{\rho_{01} \mu_{02}^{2}+\rho_{02} \mu_{01}^{2}} \cdot \frac{4 \pi \sigma}{\left(u_{01}-u_{02}\right)^{2}} .
$$

Perturbations with a wavelength smaller than $\lambda_{c}$ will die out, whereas the ones with a wavelength larger than $\lambda_{c}$ will grow exponentially. However, the instability must occur at a special set of wavelength or wave number, which is the dominant (fastest) mode instability. This dominant wavelength $\lambda_{m}$ is usually $\sqrt{3}$ times $\lambda_{c} \cdot{ }^{25}$ In fact, $\lambda_{m}$ corresponds to the ripple spacing observed finally. For the current pulse laser on Vit 1 BMG with the intensity of $\sim 10^{13} \mathrm{~W} / \mathrm{m}^{2}$, the velocity of Vit 1 melt may be taken to be zero compared to the plasma plume velocity, which is larger than $\sim 10^{3} \mathrm{~m} / \mathrm{s}^{6}{ }^{6}$ The density of Vit 1 in the liquid state $\rho_{02} \approx 6 \times 10^{3} \mathrm{~kg} / \mathrm{m}^{3},{ }^{26}$ which is much greater than that $\left(\sim 1-2 \mathrm{~kg} / \mathrm{m}^{3}\right)$ of plume. ${ }^{27}$ The Vit 1 melt has the viscosity of about $10^{1}$ Pas that is much larger than that (close to atmospheric viscosity, $10^{1} \mu$ Pas) of plume. ${ }^{28}$ Considering that $\mu_{01} \ll \mu_{02}, \rho_{01} \ll \rho_{02}$, and $u_{02} \rightarrow 0$, the ripple spacing is actually determined by the density and initial horizontal velocity of the expanding plasma for fixed $\sigma \approx 0.83 \mathrm{Nm}^{-1},{ }^{29}$ as shown in Fig. 2 . In fact, there is a trend that the plasma with lower density has the higher velocity. ${ }^{30}$
Therefore, the predicted $\lambda_{m}$ usually ranges from the corner "A" to "B," between 1 and $6 \mu \mathrm{m}$. The actually measured spacing of the surface ripples (see Fig. 1) falls nicely in this range of values. Such agreement validates the K-H instability mechanism for the observed ripples on the Vit 1 BMG by nanosecond pulse laser with single shot. The laser-induced fluid dynamics instability implies that the BMGs have the capability to even energy absorption and resultant flow upon the nanosecond pulse laser. The BMG can behave a liquid with the supply of energy even at nanosecond time scale, showing the super fluidity. This is because the BMGs have very short mean free path of electron and lack in gain boundaries ${ }^{31}$ compared to the polycrystalline alloys.

Financial support is from the NSFC (Grants Nos. 10725211, 11002144, 11021262, and 10902113), the National Natural Science Foundation of China-NSAF (Grant No. 10976100), and the National Key Basic Research Program of China (Grant No. 2009CB724401).

${ }^{1}$ T. H. Maiman, Nature 187, 493 (1960).

${ }^{2}$ J. F. Ready, Industrial Applications of Lasers, 2nd ed. (Academic, Chestnut Hill, MA, 1997).

${ }^{3}$ N. R. Isenor, Appl. Phys. Lett. 31, 148 (1977).

${ }^{4}$ R. Le Harzic, D. Dorr, D. Sauer, F. Stracke, and H. Zimmermann, Appl. Phys. Lett. 98, 211905 (2011).

${ }^{5}$ S. Lugomer, Phys. Lett. A 361, 87 (2007).

${ }^{6}$ L. K. Ang, Y. Y. Lau, R. M. Gilgenbach, H. L. Spindler, J. S. Lash, and S.

D. Kovaleski, J. Appl. Phys. 83, 4466 (1998).

${ }^{7}$ G. S. Zhou, P. M. Fauchet, and A. E. Siegman, Phys. Rev. B 26, 5366 (1982).

${ }^{8}$ M. F. Ashby and A. L. Greer, Scr. Mater. 54, 321 (2006).

${ }^{9}$ C. A. Schuh, T. C. Hufnagel, and U. Ramamurty, Acta Mater. 55, 4067 (2007).

${ }^{10}$ M. W. Chen, Annu. Rev. Mater. Res. 38, 445 (2008).

${ }^{11}$ L. H. Dai and Y. L. Bai, Int. J. Impact Eng. 35, 704 (2008).

${ }^{12}$ W. H. Wang, Adv. Mater. 21, 4524 (2009).

${ }^{13}$ W. Jia, Z. N. Peng, Z. J. Wang, X. C. Ni, and C. Y. Wang, Appl. Surf. Sci. 253, 1299 (2006).

${ }^{14}$ M. Sorescu, Phys. Rev. B 61, 14338 (2000).

${ }^{15}$ A. P. Radlinski, A. Calka, and B. Lutherdavies, Phys. Rev. Lett. 57, 3081 (1986).

${ }^{16}$ H. Sun and K. M. Flores, Metall. Mater. Trans. A 41A, 1752 (2010).

${ }^{17}$ H. Sun and K. M. Flores, J. Mater. Res. 23, 2692 (2008).

${ }^{18}$ Y. Kawahito, T. Terajima, H. Kimura, T. Kuroda, K. Nakata, S. Katayama, and A. Inoue, Mater. Sci. Eng., B 148, 105 (2008).

${ }^{19}$ D. T. A. Matthews, V. Ocelik, and J. T. M. de Hosson, Mater. Sci. Eng. A 471, 155 (2007).

${ }^{20}$ F. Audebert, R. Colaco, R. Vilar, and H. Sirkin, Scr. Mater. 48, 281 (2003).

${ }^{21}$ K. X. Liu, W. D. Liu, and L. M. Ye, J. Appl. Phys. 109, 043109 (2011).

${ }^{22}$ F. X. Ma, J. J. Yang, X. N. Zhu, C. Y. Liang, and H. S. Wang, Appl. Surf. Sci. 256, 3653 (2010).

${ }^{23}$ S. Chandrasekhar, Hydrodynamic and Hydromagnetic Stability. (Dover, Inc., New York, 1981).

${ }^{24}$ D. A. Sanchez, Ordinary Differential Equations and Stability Theory (Freeman, San Francisco, 1968).

${ }^{25}$ F. Spaepen, Acta Metall. 23, 615 (1975).

${ }^{26}$ G. J. Fan, H. H. Liao, H. Choo, P. K. Liaw, N. Mara, A. V. Sergueeva, A. K. Mukherjee, and E. J. Lavernia, Metall. Mater. Trans. A 38A, 2001 (2007).

${ }^{27}$ B. X. Wu, Appl. Phys. Lett. 93, 101104 (2008).

${ }^{28}$ R. Busch, E. Bakke, and W. L. Johnson, Acta Mater. 46, 4725 (1998).

${ }^{29}$ W. J. Wright, Doctoral thesis, Stanford University, 2003.

${ }^{30}$ P. L. G. Ventzek and R. M. Gilgenbach, J. Appl. Phys. 68, 965 (1990).

${ }^{31}$ M. Born and K. Huang, Dynamical Theory of Crystal Lattices (Oxford University Press, Oxford, 1954). 